\title{
La dinámica del dar y recibir: El caso Ángela a la luz del concepto de Mendicidad de E.Ghent ${ }^{1}$
}

\section{Mercedes Güell² \\ Barcelona}

\begin{abstract}
Este trabajo es una reflexión sobre cómo la lectura del escrito de Peter Shabad: "Resentimiento, indignación y derecho; la transformación del deseo inconsciente en necesidad", y el comentario de Emmanuel Ghent a dicho texto en el que desarrolla el concepto de Mendicidad, no solo iluminó y transformó el trabajo terapéutico con mi paciente Ángela sino que me ayudó a profundizar en las dinámicas del Dar y recibir que se ponen en juego en cualquier interacción entre 2 personas.
\end{abstract}

Palabras clave: Mendicidad, Necesidad, Reconocimiento, Dar y recibir, Responsabilidad, Empatía, Vergüenza

This paper is a reflection on how reading the paper of Peter Shabad: "Resentiment, indignation, Entitlement; the transformation of Unconscious Wish into Need "and the commentary by Emmanuel Ghent to the text in which develops the concept of Begging, not only illuminated and transformed the therapeutic work with my patient Angela but helped me to delve into the dynamics of Dar and receive that come into play in any interaction between 2 people.

Key Words: Begging, Need, Recognition, Giving and Receiving, Responsibility, Empathy, Embarrassment.

English Title: The dynamics of giving and receiving: Angela's case in light of the concept of Begging by E. Ghent

Cita bibliográfica / Reference citation:

Güell, M. (2016). La dinámica del dar y recibir: El caso Ángela a la luz del concepto de Mendicidad de E.Ghent. Clínica e Investigación Relacional, 10 (1): 113-124. [ISSN 1988-2939] [Recuperado de www.ceir.info ] DOI: 10.21110/19882939.2016.100109

\footnotetext{
1 Texto presentado en la 6a Reunión anual de IARPP-España, Valencia, Octubre 2015

2 Miembro de IARPP-España, Barcelona. 
"La expresión directa de un deseo subyacente de apoyo y protección conlleva una calidad espontánea. Tales experiencias de necesidad se experimentan por los otros como no coercitivas y tienden a elicitar de forma natural la protección que se busca. La mendicidad por otra parte, se conceptuaría como una respuesta del self, una respuesta a la propia autocrítica y expectativa de abandono" (E.Ghent).

\section{INTRODUCCION}

Esta comunicación es una reflexión sobre cómo la lectura del artículo de Peter Shabad "Resentiment, Indignation, entitlement; The transformation of unconscious wish into need", y el comentario de Emmanuel Ghent a dicho texto en el que desarrolla el concepto de Mendicidad, no solo iluminó y transformó el proceso terapéutico con mi paciente Ángela sino que me ayudó a profundizar en la dinámica del dar y recibir que se pone en juego en cualquier interacción.

El texto de P. Shabad expone de forma muy sugerente las situaciones de "impasse" que se crean en terapia cuando el estado de necesidad y la posición de demanda son expresados por el paciente desde la rabia, el resentimiento y la queja.

Cuando un individuo cuenta con una historia evolutiva en la que las necesidades instintivas de protección y amor han sido respondidas con abandonos y castigos, el individuo se convierte en el principal crítico de sus propias necesidades legítimas y las disocia en diversos grados. De esto puede derivarse que le sea imposible expresar sus necesidades directamente y así perpetuar un patrón relacional en el que dichas necesidades no se satisfagan. En esta situación se recurre a menudo al resentimiento y la indignación no solo como consecuencia de la privación inicial sino por el fracaso continuo de los otros en satisfacer nuestras carencias experimentando una sensación de merecimiento, de derecho a recibir alguna compensación por lo no recibido. Algunos de los impasses terapéuticos más difíciles se derivan de la puesta en escena de dicho patrón relacional (P. Shabad).

Donde Shabad nos habla de "deseo", Ghent nos habla de "necesidad" y lo que para Shabad es considerado necesidad, para Ghent sería un estado de mendicidad. La diferenciación de estos conceptos no es fácil de esclarecer. Utilizaré los conceptos de deseo y necesidad de forma indistinta y me referiré al concepto de Mendicidad de Ghent como aquella forma en la que una persona expresa y formula lo que necesita desde la rabia y el resentimiento: "

"La expresión directa de un deseo subyacente de apoyo y protección conlleva una calidad espontánea. Tales expresiones de necesidad son experimentadas por los otros como no coercitivas y tienden a elicitar de forma natural la protección que se busca. La Mendicidad por otra parte, se conceptuaría como una respuesta del self, una respuesta a la propia autocrítica y expectativa de abandono" (J. Safran). La expresión de Mendicidad tiende a ser experimentada por el otro como demandante, coercitiva e imposible de satisfacer. 
En nuestro trabajo nos encontramos a menudo con situaciones en las que nuestra dedicación y entrega, nuestras "buenas intenciones" de comprender y ayudar a nuestros pacientes no solo caen en "saco roto, sino que pueden llegar a producir en ellos un mayor desamparo y un mayor sentimiento de vacío y abandono. La confianza en la bondad de la empatía queda cuestionada en esos momentos.

Mi impotencia y mi preocupación ante estas experiencias en terapia y las dificultades con las que me encontré en el caso de Ángela que más abajo desarrollaré, me han llevado explorar y a buscar respuestas en los textos a los que hago referencia, así como en los espacios de supervisión y trabajo personal.

"El paciente puede experimentar la empatía comprensiva del terapeuta como una forma de taponar o cerrar su resentimiento. A menudo se puede estar tan lleno de rabia que se boicotee de forma inconsciente el deseo de ser comprendido. Se entra entonces en una espiral de constante sabotaje y rechazo a lo que precisamente se está buscando satisfacer". Para el paciente que ha crecido en un mundo de ambivalencias, en donde los otros son vividos como alguien peligroso y falso, el ofrecimiento de empatía y comprensión puede ser vivido como algo sospechoso.

El paciente puede ser tan insistentemente demandante hacia el otro que puede permanecer ciego a los intentos del terapeuta de ofrecer voluntariamente cuidado y comprensión. Continúan demandando convencidos de que no van a recibir lo que necesitan.

La expresión "Si lo pides así, no te lo voy a dar. Pídelo bien", que probablemente hemos escuchado alguna vez como hijos e incluso hemos dicho como padres, refleja muy bien esta situación de conflicto entre lo que se necesita y no puede ser recibido por ser expresado de forma brusca y desesperada. Como cuando abrimos los brazos a alguien con tanto anhelo y tanta rabia a la vez por no ser atendidos cuando lo necesitamos, que entonces cuando por fin llega la ayuda, sentimos que ya no la queremos y cerramos los brazos como muestra de nuestro dolor.

En terapia, nos dice Shabad encontramos individuos que han experimentado mucho dolor psíquico en los que la expresión de necesidad de forma resentida y compulsiva (el estado de mendicidad de Ghent) se convierte en la única forma de comunicar su dolor, ya que refiriéndose a uno mismo como alguien necesitado, dominado por la frustración y el vacío, se puede evitar la cruel crítica que uno imagina recibiría de los otros por el solo hecho de desear y anhelar la comprensión y el amor. Detrás de la mirada autocrítica y la vergüenza no confesada nos protegemos así del dolor que experimentaríamos al no recibir del otro aquello que es legítimo y valioso per se.

Cómo transformamos esta espiral destructiva y estéril ¿Cómo llegamos a comprender este dolor profundo sin ser arrastrados por él? ¿Gratificamos al paciente convirtiéndonos esta vez en las figuras protectoras y validantes que no tuvo? ¿es así de fácil? ¿Por qué el paciente no puede recibir nuestra ayuda libremente? ¿Qué hay de nuestra historia personal como terapeutas en todo esto? 
Shabd no cuestiona abiertamente el estado de "buenismo" de un ambiente sostenedor como el que defiende Winicott, ni la bondad de proveer al paciente de nuestra total entrega ya que señala como algo fundamental crear este ambiente en el vínculo, pero cree que hay cuestiones cruciales de la responsabilidad de uno mismo por su terapia y su vida que están en juego. Al animar a los pacientes a articular los significados subjetivos que sus experiencias de necesidad y sus demandas tienen para ellos vemos cómo aparecen la desilusión por los antiguos deseos anhelados y un amargo resentimiento por una infancia que no puede recuperarse y la sensación del derecho legítimo a ser compensado por todas esas pérdidas.

Dicho sentimiento de sentirse con derecho puede ser activado en la transferencia a través de la dimensión de pasividad-actividad, en donde el peso de la responsabilidad del resultado del tratamiento se proyecta sobre el terapeuta. Si esta situación se topa con la tendencia a reparar y dar de forma urgente por parte del terapeuta, se hará muy difícil salir del impasse terapéutico. Shabad señala que esta dinámica sólo se puede revertir si no contraatacamos con irritación, al tratar de compartir la responsabilidad conjunta hacia el proceso de la cura.

¿Cómo entender esta responsabilidad?, ¿qué parte nos toca en el establecimiento del impasse terapéutico?

\section{EL CASO ANGELA:}

"Tu comprensión no me ayuda, no me entra lo que me dices, no me sirve."

Cuando Ángela me escribió estas palabras en un mail llevábamos 2 años y medio de terapia.

Tenía 23 años y un largo recorrido por diferentes terapias y centros de día debido a un diagnóstico de trastorno de alimentación acompañado de síntomas de ansiedad persistente, alteraciones en el sueño y falta de concentración.

Empezamos con una pauta de 2 sesiones semanales. El sentimiento de ansiedad y angustia llenaba la hora entera de las sesiones. Expresaba su angustia y su impotencia de forma muy poco elaborada: "estoy mal, no me encuentro bien, no puedo, tengo ansiedad", eran sus frases constantes, repetidas semana tras semana. Mi intento de poner palabras a su sufrimiento, de significarlo, de ayudarla a expresar su malestar para entenderlo, de poder nombrar lo que necesitaba, no sólo no servía sino que parecía desanimarla aún más.

Cuando Ángela llegó a mi consulta estaba trabajando como auxiliar de cocina y había comenzado a preparar un examen de acceso para poder matricularse en un grado superior de nutrición. La ilusión y el anhelo por alcanzar sus objetivos se veía frustrado y bloqueado por ese estado de ansiedad constante, una angustia por no poder concentrarse y un miedo atroz a suspender que me expresaba en las sesiones con una mirada triste y desesperanzada a la vez.

Tenía esperanzas en una terapia que la sacara de ahí, que la ayudara a poder concentrarse y llevar a cabo sus proyectos pero todo era vivido como un reto imposible y una auto exigencia desmedida que le impedían avanzar y manejar las dificultades. Las distintas terapias que 
había probado siempre acababan decepcionándola, dejándola con un sentimiento de que nadie podía ayudarla.

El temor a "no poder conseguirlo" comenzó a provocar boicots constantes a sus planes de estudio y a sus compromisos; en pocos meses cambió de trabajo y de escuela. Le invadía un sentimiento muy doloroso de no encajar en ningún sitio, de no sentirse cómoda en los grupos.

Ángela sufría mucho al sentirse exageradamente dependiente de su pareja y sentía mucha rabia hacia sus padres y su hermana por lo que ella vivía como una falta absoluta de comprensión. Estaba permanentemente enfadada con todo.

El vínculo terapéutico empezó a construirse en base a una demanda compulsiva por su parte y una disponibilidad casi absoluta por la mía; mensajes al móvil a cualquier hora del día, cambios en las horas de las sesiones acordadas, anulaciones. Yo accedía y me adaptaba a sus demandas algo confusa pero intuyendo que esa era la única forma que entonces teníamos de relacionarnos.

Desde el principio de nuestro trabajo juntas sus quejas supusieron el principal obstáculo para avanzar. Yo sabía que era la única forma que ella tenía de expresar su dolor pero no conseguíamos transformarlas, de alguna forma también a mí me paralizaban:

"no avanzo, sigo igual, siento un agujero, un vacío horrible", me decía mientras me señalaba la boca del estómago. Quiero solucionar mis problemas no darles vueltas, trabajarlos de forma más directa, tanta explicación me agota. Yo observaba sus ojeras por haber dormido mal, su tez blanca, casi gris y me preguntaba por qué las sesiones no tenían un efecto calmante, no le proporcionaban alivio alguno.

Hablamos de lo que le preocupaba, intentábamos entender, recorrimos su historia y sus experiencias infantiles tratando de crear puentes y nuevas formas de relacionarse $y$ enfrentarse a sus dificultades.

Cuando Ángela tenía 8 años su madre pasó por un periodo de depresión que hasta ahora era desconocido para ella. Ángela recuerda pasar mucho miedo por las noches y terror cada vez que tenía que separase de ella. Su memoria describe una niña muy alegre pero enormemente inquieta y dispersa, con gran dificultad para relajarse y concentrarse. En su etapa escolar, alrededor de los 13 años Ángela sufrió bullying por parte de sus compañeros de clase, experiencia que la marcó profundamente y dejó un poso de inseguridad y fala de confianza en sí misma que todavía hoy sigue presente. Se recuerda triste e insegura pero jamás se le ocurrió contarlo en casa. El temor de una niña acosada e insegura debió caer como una losa sobre una madre triste y un padre desbordado que tuvo que hacerse cargo, como pudo, de una situación familiar muy compleja y dolorosa.

Ángela me pedía respuestas concretas y opiniones sobre las diferentes decisiones que iba tomando en su vida a pesar de que por otro lado luchaba por deshacerse del sentimiento de que su padre sabía, mejor que ella, lo que tenía que hacer o era recomendable para ella. Al 
demandarme que de alguna forma la guiara se repetía un patrón aprendido del que, por otra parte, quería desprenderse para sentirse más autónoma.

Me pedía ayuda pero si yo se la ofrecía se sentía incómoda, incompetente, no le servía; pero si no se la daba y no respondía a sus demandas me vivía como alguien insuficiente para ella y se sentía abandonada. Un mensaje que me escribió refleja bien esa ambivalencia, este nudo en el que estábamos atrapadas: "dudo de la eficacia de la terapia porque tengo la sensación de que en las sesiones intentas rescatarme y eso lo debo hacer yo. Tratas de quitarme de la cabeza mis problemas y el sentimiento de culpa que me oprime pero eso es auto engañarme. Intentas suavizar todo y poner palabras diferentes a lo que no funciona y eso me hace sentir estancada. Te sobrepasas cuando me ves mal y cuando noto que no sabes qué decirme y no haces nada, me siento desprotegida"

Mi agotamiento empezó a hacerse visible. Mis preguntas en el espacio de supervisión y en mi propia terapia empezaron a girar en torno a cómo manejar mi impotencia y mi enfado. Puse el foco en mis sentimientos y concentré mis esfuerzos en mi afecto en la contratrasferencia para encontrar una salida a la tensión que sentíamos las 2.

¿Qué me pedía Ángela detrás de su queja? ¿Me pedía algo, pero no podía recibirlo? ¿Qué sentía yo? ¿Desde dónde respondía yo a su demanda?

\section{DE LA IMPOTENCIA A LA REFLEXION SOBRE MI AFECTO EN LA CONTRA TRANSFERENCIA COMO ELEMENTO LIBERADOR}

"Podemos considerar la contratransferencia como una forma a través de la cual el analista trata de llegar al paciente, de alcanzarlo" (D.B. Stern).

Las reflexiones de varios autores sobre este tema me han servido de guía y han sido una preciosa fuente de inspiración para seguir avanzando en mi trabajo. ¿Cómo usar "las heridas del terapeuta" de las que nos habla D. Wallin, como herramienta para salir del impasse?

Mitchell nos ayuda a ser conscientes de la dificultad de entrar a reflexionar sobre la contratransferencia: "prestar atención a la contratransferencia, de acuerdo, pero qué hacemos con ella; ¿Interpretamos o no interpretamos? ¿Revelamos o no revelamos? ¿Nos expresamos o mantenemos la reserva? ¿Cómo y desde dónde decidimos hacer lo que hacemos?

¿Lo que me pasa está relacionado con material del paciente o me aleja de él? ¿Mi sentimiento actual puede serle útil al paciente o puede perjudicarle?

¿Las palabras que formulo, como dice D.B. Stern, arrojan luz sobre las zonas de experiencia no formulada o las oscurecen y amortiguan?

La experiencia del analista, sigue Mitchell, puede verse afectada por los mismos afectos, dinámicas y conflictos que está intentando que el paciente entienda como propios: 
Me di cuenta que yo estaba respondiendo a los afectos que Ángela traía a las sesiones también desde la urgencia y la rabia. Estaba empeñada en sacarla de su vacío, en colmarlo con ofrecimientos de ayuda y comprensión, pero al hacerlo así me alejaba de ella porque me sentía incompetente e insuficiente al no recibir la respuesta esperada y, de esa forma, me apartaba de ella y de la posibilidad de comprenderla. Mis emociones reactivas dificultaban el encuentro entre nuestras dos mentes. No la podía ver, solo necesitaba que mejorase para encontrar sentido al esfuerzo de mi trabajo con ella y dejar de dudar de mi capacidad para ayudarla.

El locus central del cambio terapéutico, se encuentra, para Mitchell, en el esfuerzo del analista por encontrar una nueva manera de participar dentro de sí mismo y luego con el paciente. Esto es muy diferente a dar por supuesto un nuevo crecimiento allí donde pueden estar dándose sutiles formas de repetición".

En nuestra terapia se repetían y entrelazaban los distintos patrones que cada una aprendió en su infancia. Ángela no tenía confianza en sus decisiones y su percepción sobre las cosas. Su padre ocupaba el lugar del "que sabe". Ese lugar lo ocupaba yo sin ser del todo consciente desde mi patrón aprendido de hacerme responsable del otro en la relación de forma unilateral, y Ángela se revelaba desde la irritación. Con la embestida a mi trabajo y mi capacidad ella intentaba revertir lo aprendido y a mí me costaba ver en ello su búsqueda de autonomía y su profundo deseo de confiar en ella misma y ser apoyada.

Elisabeth Bishop reflexiona sobre las sutiles pero cruciales diferencias en la forma en la que los dones del analista son experimentados y en los sentimientos del donante sobre lo que ofrece.

Pensar todo esto me ha ayudado a darme cuenta de que no se trataba de ofrecerme en entera disponibilidad ni tampoco de frustrar su demanda para que ella pudiera conectar con sus emociones y afectos y reconocerme a mí como alguien diferente que trataba de ayudarla. Tenía más que ver con un equilibrio muy preciso, una línea fina que se ha ido construyendo poco a poco a partir de la restauración de la dinámica del dar y recibir.

Ha sido el reconocimiento de lo que se ha movido en mí todo este tiempo: mi propia impotencia, la rabia, mi rol de cuidador ineficaz, mi sentimiento de ser la única responsable de la relación, el peso que eso conlleva y mi dificultad para pedir y recibir, lo que ha permitido empezar a reconocernos, a sentirnos al mismo nivel, a entender y abrirnos a la posibilidad de hacernos más responsables las dos de nuestras acciones y nuestras emociones, asumiendo el efecto que dichas emociones y acciones tienen sobre los demás.

Al contario que la experiencia del deseo, que suele comunicar de forma implícita una solicitud de respuesta en el otro, la experiencia de necesidad (el estado de mendicidad), con su implicación como demanda, puede dañar y distorsionar el libre discurso del dar y recibir, (Shabad). 


\section{LA DINAMICA DEL DAR Y RECIBIR: CONSTRUCCIÓN DE LA MUTUALIDAD Y EL RECONOCIMIENTO.}

Atwood subraya que lo que las personas nos muestran depende, en gran parte, de cómo nosotros estamos respondiéndoles. La experiencia humana siempre está inmersa en un contexto relacional. Una idea nueva apareció en mis reflexiones dentro de mi propia terapia personal: ¿Es posible que, de alguna manera, la mendicidad sea contra-transferencial? ¿Podía cambiar mi mirada sobre Ángela y sobre mí de forma que surgiera algo nuevo?

El momento que quiero describir y que marcó un punto de inflexión en nuestro trabajo tuvo lugar tras un período de desconexión y alejamiento de las sesiones. Yo sabía que Ángela había estado buscando otros profesionales que pudieran ayudarla mejor. Lo habíamos compartido en las últimas sesiones y yo, algo indecisa, le animé a que probara diferentes enfoques terapéuticos. Creo que detrás de mi compromiso por validar su iniciativa se encontraba también cierto alivio y descanso. Aun así sabía que habíamos recorrido un camino valioso y podía reconocer mi esfuerzo en la terapia gracias al cual pude, en muchos momentos, apoyarme para continuar.

Tras varios meses sin venir a la consulta, en los que siguió comunicándose conmigo vía mail o por mensajes en el móvil preguntándome mi opinión sobre sus cambios y sus decisiones, Ángela me llamó y pidió una hora de visita:

Llegó a la consulta con una expresión diferente de la habitual, nunca antes había llorado en las sesiones. A pesar de sentir que era una persona muy cariñosa siempre traía una mueca de disgusto, de cansancio; una mueca a veces fría y poco afectiva. Ese día tenía un nudo en la garganta cuando me dijo: "no hay nada que hacer Mercedes, nunca lo voy a conseguir, no puedo ser feliz". Noté que estaba a punto de ponerse a llorar, se mordía el labio para no hacerlo y casi no podía mirarme a los ojos. Yo sólo puede decirle: "me doy cuenta Ángela de cuánto estás sufriendo". "No quiero llorar", me dijo, "me da mucha vergüenza que me vean asi". La vergüenza entraba por primera vez en la terapia y algo nuevo comenzó a moverse dentro de mí. Me quedé callada, no dije nada. El mismo nudo en la garganta me impedía ir a socorrerla pero fui consciente de que, por primera vez, me sentía de verdad a su lado. Hoy sé que fue eso lo que permitió la conexión.

Rosa Velasco subraya que el proceso de transformar afectos, muchas veces, atraviesa por la vergüenza: "Una experiencia emocionada, nos dice, produce la disminución de aspectos escindidos o disociados de nuestra personalidad"

La vergüenza confesada nos permite salir de la vergüenza que paraliza y nos oculta. El hecho de poder comunicar este sentimiento, de no guardarlo y dejarnos ver, genera comprensión y una mayor cercanía. Posibilita una nueva mirada.

Ángela se enfadaba, se quejaba pero esa tarde, por primera vez, a través de la vergüenza y ese nudo en la garganta, me mostró su sufrimiento y yo lo pude reconocer y acompañar realmente. Sentí una gran ternura por las dos y por el esfuerzo que habíamos hecho a lo largo de todo este tiempo. Noté que mi respuesta era genuina porque no tenía prisa, ni me notaba 
angustiada. Me di cuenta de que hasta entonces yo había respondido a sus demandas y sus quejas desde la urgencia y la confusión y eso había generado en ella desconsuelo y frustración.

Creo que Ángela sintió mi estado y pudo recogerlo porque a la sesión siguiente me trajo unas galletas que me había comprado para merendar y "darme fuerzas" como dijo, y me habló de lo difícil que le resultaba tolerar las dificultades y limitaciones de los demás.

Pudimos a partir de entonces, con paciencia y más serenidad, hablar de todo ello. Ángela aceptó mi interpretación de que me parecía que al cuestionarme a mí y a la terapia ella estaba intentando hacerse responsable de sí misma y mostraba coraje y valentía pero que yo sentía, le dije, que teníamos que encontrar formas nuevas donde cupiéramos las dos y pudiéramos escucharnos, porque si no al final se acabaría sintiendo de nuevo sola e incomprendida.

Desde entonces se ha generado una forma nueva de relacionarnos. Hay un cuidado y una aceptación del espacio y las limitaciones del otro que nos permite movernos con mucha mayor confianza y libertad. $Y$ a pesar de saber ambas del camino que queda por recorrer, yo puedo ofrecerle mi dedicación y ella puede recibirla, a la vez que siente con sorpresa que puede darme cosas y yo las puedo recibir.

Hoffman en su bello artículo "Forjando la diferencia a partir de la Semejanza" nos dice que la desintoxicación del deseo requiere de la debilitación de una dicotomía en la cual el deseo de una persona excluye el de la otra. La autonomía y la receptividad emergen como complementarias en lugar de como exclusivas. Podemos empezar a hacer algo por nosotros mismos a la vez que recogemos lo que el otro nos ofrece y somos receptivos a sus sugerencias y cuidados.

El reconocimiento no es una palabra vacía, un gesto que creemos aprendido, a veces lo damos por hecho cuando realmente no está siendo sincero de verdad. No lo sabemos pero es así. Sólo nos damos cuenta cuando sentimos algo diferente, una emoción profunda ante el sufrimiento del otro que nos asegura el sentimiento de proximidad y respeto.

J. Benjamin nos dice: "La estructura del más allá del que hace y al que le hacen dificulta la agencia, haciendo que uno se sienta impotente; uno se siente reactivo en lugar de libre para dar y recibir, se siente culpable en lugar de responsable, controlado en lugar de reconocido".

La habilidad para reconocer los sentimientos del otro, así como la agencia de uno mismo se basa en la restauración del tercero y en sobrevivir a la ruptura mediante el reconocimiento mutuo

Dar nos hace bien y hace bien al otro cuando puede recibir. Recibir nos hace bien y hace bien al otro porque le permite dar. Cuando la dinámica del dar y recibir fluye libremente en las dos direcciones, se construye un espacio de mutuo reconocimiento donde podemos crecer. 


\section{LA MIRADA HACIA EL MENDIGO O EL MENDIGO QUE NOS MIRA: "UN LUGAR EN EL MUNDO"}

La mirada del mendigo nos interpela. A menudo sentimos malestar porque nos recuerda la exclusión que padecen, incluso sentimos rabia de que no puedan cambiar su situación, no nos gusta ser sólo compasivos y encontrarnos por encima de alguien, en un lugar de poder que no deseamos pero que a menudo contribuimos a establecer.

En la acera de una calle muy céntrica de mi ciudad está instalada una persona muy especial. Llevo viéndola bastantes años y siempre se coloca en la misma esquina, con el mismo pañuelo en el suelo sobre el que tiene esparcidas pequeñas piedras de distintos colores junto a una cesta para recoger el dinero que la gente le deja al pasar. Muestra su torso desnudo con una deformidad en la espalda fruto, imagino, de alguna malformación genética o algún trágico accidente. Es minusválido. Sus piernas son cortas, delgadas y asimétricas y están colocadas como dos ramas entrelazadas en postura de meditación. La última tarde que me acerqué a su esquina yo ya estaba preparando esta comunicación y supongo que se me hizo patente algo que yo llevaba años sintiendo. Le di una moneda y el me ofreció una piedra azul. Siempre conversamos un poco y le dije medio en broma: "azul ya tengo, ¿por qué no me das esa verde que tienes al lado? Me miró sonriendo con sus ojos azules y una mirada confiada y serena y me dijo:" verdes me quedan pocas, tu vienes a menudo y tengo que guardármelas para alguien más caprichoso". Yo también sonreí.

No vende sus piedras, las ofrece si le parece bien o quiere hacerlo. A veces he visto que según a qué persona y no sé con qué criterio, no entrega sus piedras. Las ofrece como intercambio cuando siente que así tiene que ser. Quiero pensar que es cuando siente que el otro las va a valorar.

En su forma de pedir no hay mendicidad, ni rabia, ni una mirada de rencor. No expresa un sentimiento de sentirse víctima a pesar de que la vida se lo ha puesto muy difícil. Hay una dignidad poderosa y firme en su forma de estar en el mundo que permite que la dinámica del dar y recibir que en cualquier caso parecido pudiera obedecer a relaciones de poder y desigualdad, en su caso no es así. Él ha encontrado un lugar en el mundo desde el cual puede dar y recibir sintiéndose parte de la comunidad.

Es muy interesante cómo $P$. Shabad en su artículo reflexiona, alrededor de cómo la expresión de deseos y necesidades tiene diferentes implicaciones según el tipo de relación psicoterapéutica que se establece, así como el tipo de contrato social implícito que gobierna el intercambio de dar y recibir.

¿Dónde nos lleva y nos coloca esto como psicoterapeutas?

Después de todo, nos dice, nuestras respectivas visiones de lo que constituye una terapia, así como nuestro consecuente uso de la relación terapéutica para llevar a cabo la cura, llevan implícitos supuestos y asunciones no articuladas de lo que es oportuno y adecuado que una persona pueda esperar recibir por parte del otro. Así la forma en la que decidimos participar en la relación terapéutica refleja y a la vez crea (con todo el poder hipnótico de la 
transferencia y la contratransferencia) un lazo que interviene e influye en el contrato social que gobierna el intercambio del dar y recibir.

Para Shabad el lenguaje de las "necesidades" (no de los deseos), refleja, por lo menos en parte, la influencia prolífica de la psicoterapia clásica y, específicamente, de la relación unilateral entre paciente y terapeuta: "parece que se ha convertido en una tendencia inconsciente, el hecho de designar las relaciones como aquellas en las que o bien se es una parte necesitada que recibe, o bien un dador compulsivo de cuidados empáticos. Esta clase de movimiento rígido de una a otra parte, entre recibir y dar, contribuye en buena medida a una cultura del narcisismo, constituida por una cadena humana de necesidades y demandas por un lado y cuidados bajo mandato por otra".

Rank nos ayuda a pensar que la dificultad de poder crear un vínculo auténticamente recíproco puede tener que ver con el hecho de que ser un agente creativo y apreciar plenamente nuestras responsabilidades como fuente de influencia sobre el mundo y los demás, es quizás un lugar que el ser humano esquiva por temor a la perspectiva de ser totalmente dueño de su vida.

La aceptación de nuestros afectos en la contra-transferencia junto al reconocimiento de la responsabilidad de nuestra participación en la terapia, contribuye creo, de forma muy significativa, a convertir la escena terapéutica en un lugar privilegiado para un aprendizaje de la igualdad y la humildad.

Esto es lo que mi paciente Ángela me ha ayudado a entender.

\section{SIEMPRE PODEMOS ENCONTRAR NUDOS DONDE ATAR NUEVOS MUNDOS (Verónica Greber)}

"Todo lo que hemos logrado en análisis se ha construido, parcialmente, sobre fuerzas que el paciente traía consigo y que antecedian a la relación terapéutica (Hoffman)

El interés que compartimos por el cine es el espacio potencial de creatividad donde el trabajo con Ángela se relaja y nos podemos sentir cómodas. Compartimos películas que comentamos, alabamos o criticamos sin sentir que una sabe más que la otra. Su capacidad reflexiva aumenta y se vuelva más rica, su inteligencia se despliega, la tensión disminuye y aparecen referencias y asociaciones entre los personajes y sus vivencias que nos ayudan a profundizar sobre sí misma y sus experiencias.

Nuestra pasión compartida fue el puente que encontramos para abrirnos paso más allá del vacío que, a menudo, dejaban las palabras.

\section{CONCLUSIONES}

"puesto que aprender a hacer este trabajo es un asunto a menudo tan torturante, tan intenso, tan cargado de responsabilidad, confusión y temor, nos esforzamos mucho por 
cultivar una versión del self que podemos reconocer como profesional, analítica y competente, pero los momentos y períodos más interesantes y productivos del trabajo terapéutico son, con frecuencia, precisamente aquellos que ocurren fuera del self profesional reconocible y reasegurador, tiempos en los que la confusión, el temor, la excitación, la exasperación, el anhelo o la pasión son los afectos dominantes. (Mitchell)

Reflexionar sobre mis afectos en la contratransferencia es la mejor vía que he encontrado para profundizar y enriquecerme de esos momentos de los que habla Mitchell.

En mi trabajo con Ángela pasé por momentos de enorme desconcierto y tensión. El enfado me protegía como también la protegía a ella.

Mi patrón aprendido, mi forma de estar en el mundo chocó con la de mi paciente y estuvimos a punto de fracasar. Me di cuenta de que no sufría con ella, más bien sufría contra ella y pude sentir cómo las secuelas de mi propia historia personal resonaban de nuevo en el espacio entre sesiones bloqueando mi creatividad y mi confianza.

Fue el trabajo con estos textos junto al apoyo de mis espacios de cuidado lo que me permitió salir de ahí y restaurar la dinámica del dar y recibir que se encontraba totalmente bloqueada Hoy hay más en Ángela y más en mí, modos nuevos de construir y entender nuestras experiencias $y$, nuestros afectos.

\section{REFERENCIAS}

Benjamin, J. (2012). El tercero. Reconocimiento (texto presentado en el congreso de Tel Aviv y en la $3^{\text {a }}$ reunión anual de IARPP España, 2012)).

Ghent, E. (1993). Wish, Need, and neediness commentary on Shabad's "Resentiment, indignation, entitlement. Psychoanalytic Dialogues 3 (4)

Hoffman (2007). Forjando la Diferencia a partir de la Semejanza: La multiplicidad de la experiencia correctiva. Aperturas Psicoanalíticas, No 26

Mitchell, S. (2014). Influencia y autonomía en Psicoanálisis. Madrid: Ágora Relacional

Safran, J.D (2000). La Alianza terapéutica, Una guía para el tratamiento relacional. Bilbao: Desclée. 2005

Shabad,P . (1991). Resentiment, indignation, Entitlement; the transformation of Unconscious Wish into Need. Psychoanalytic Dialogues 3(4)

Velasco, R (Feb, 2009). Dismorfofobia o vergüenza del cuerpo. Revista CelR Vol 4(1)

Wallin, D (2014) "Las heridas del terapeuta como herramientas para la terapia". Artículo traducido por un grupo de estudio en Barcelona.

Original recibido con fecha: 24/10/2015 Revisado: 20/12/2015 Aceptado: 28/02/2016 